\title{
A Pediatrics-Based Instrument for Assessing Resident Education in Evidence-Based Practice
}

\author{
Lauren Chernick, MD; Martin Pusic, MD, MA; Heather Liu, MD; \\ Hector Vazquez, MD, MSc; Maria Kwok, MD, MPH
}

Objective.-The principles of evidence-based practice (EBP) are a mandated component of the pediatric residency curriculum; however, a pediatrics-based assessment tool validated with pediatric residents does not exist.

Methods.-We designed an assessment instrument composed of items in 4 categories: 1) demographics; 2) comfort level; 3) selfreported practice of EBP; and 4) EBP knowledge. This last section required participants to identify best evidence and most appropriate study design by using pediatric-based scenarios, develop searchable questions, and use existing published research to address diagnostic and treatment issues. Four groups completed the instrument: preclinical medical students (MS-2), incoming pediatric interns (PGY-1), incoming second- and third-year pediatric residents (PGY2-3), and expert tutors (expert). We determined internal consistency, interrater reliability, content validity, item difficulty, and construct validity.

Results.-Fifty-six subjects completed tests (MS-2, n $=13$; PGY-1, $\mathrm{n}=13$; PGY2-3, $\mathrm{n}=22$; expert, $\mathrm{n}=8$ ). Internal reliability was good, with Cronbach's $\alpha=.80$. Interrater reliability was high $(\kappa=0.94)$. Items were free of floor or ceiling effects. Comfort level and self-reported practice of EBP increased with expertise level and prior EBP experience $(P<.01)$. Scores on the knowledge section (out of $50 \pm \mathrm{SD}$ ) rose with training level (MS-2: $14.8 \pm$ 5.7; PGY-1: $22.2 \pm 3.4$; PGY2-3: $31.7 \pm 6.1$; experts: $43 \pm 4.0 ; P<.01)$. Scores also correlated with prior EBP education.

Conclusions.-We have developed a reliable and valid instrument to assess knowledge and skill in EBP taught to pediatric residents. This instrument can aid pediatric educators in monitoring the impact of the EBP curriculum.

KEY WORDS: evidence-based medicine; evidence-based practice; pediatrics; postgraduate medical education; undergraduate medical education

Academic Pediatrics 2010;10:260-5
$\mathrm{E}$ vidence-based practice (EBP) refers to the use of the current best evidence in making decisions about the care of individual patients. ${ }^{1}$ In the last 2 decades, the teaching and practice of EBP have risen to high priority in the academic medical community and in the field of pediatrics. $^{2}$ The Accreditation Council for Graduate Medical Education (ACGME) recognizes the importance of EBP and the need for residents to be able to investigate and evaluate their patient care practices, appraise and assimilate scientific evidence, and improve their patient care practices (the practice-based learning and improvement competency). ${ }^{3}$ The Academic Pediatric Association also encourages pediatric residency programs to focus on the content and skills of EBP. ${ }^{4}$

As pediatric educators implement training in EBP, they need instruments to evaluate the impact of these new curricula. ${ }^{5}$ In a 2006 systematic review, Shaneyfelt and colleagues $^{5}$ identified 104 unique reports of instruments evaluating EBP where there was sufficient description to permit analysis and quantitative results. Only half of the

From the Department of Pediatrics, Division of Pediatric Emergency Medicine, Columbia University, New York, NY.

Address correspondence to Martin Pusic, MD, MA, Columbia University, Division of Pediatric Emergency Medicine, Department of Pediatrics, PH1-137, 622 W168th St, New York, New York 10032 (e-mail: mvp19@ columbia.edu).

Received for publication April 6, 2009; accepted March 27, 2010. instruments demonstrated at least 1 type of validity, whereas very few instruments documented multiple forms of validity evidence. No instrument validated with pediatric residents established multiple forms of validity. Specifically, the Berlin and Fresno questionnaires were noted to be the instruments that evaluated all components of EBP knowledge, but even those instruments are not ideal. $^{5,6}$ The Berlin questionnaire restricts assessment to EBP applied knowledge. The Fresno questionnaire requires considerable time and expertise to grade. Increasing scores on the Berlin and Fresno examinations did not differentiate amongst levels of resident learners. ${ }^{5-7}$

Pediatric educators who implement EBP curricula need instruments to document the EBP ability of individual trainees and to assess the success of new curricula. Cognitive science suggests that the context in which information is learned plays a role in its accessibility. ${ }^{8}$ It would follow that an assessment instrument whose clinical examples are true to a residents' actual practice can result in more accurate assessment in which less effort is devoted to decoding the clinical information and more effort is devoted to the EBP principles. In the review of Shaneyfelt and colleagues, ${ }^{5}$ we note that there were multiple examples of instruments for all of the major surgical and medical specialties except for pediatrics, where there was only 1 validated with pediatric residents. Our aim was to develop and validate an instrument based on pediatric content for assessing EBP knowledge of pediatric residents. 


\section{METHODS}

\section{Setting}

The development and validation of the instrument was carried out in a large academic hospital. The institutional review board at Columbia University approved the study. The pediatric residency program is composed of 20 residents a year, totaling 60 residents in all. Approximately $25 \%$ of these residents are male. The EBP curriculum in our residency program uses the Users' Guides to the Medical Literature: A Manual of Evidence-Based Clinical Practice as the core text. ${ }^{9}$ Pediatric residents receive a weekly teaching conference emphasizing key EBP concepts, including how to properly evaluate published articles. Third-year residents are also taught to incorporate EBP into their "grand rounds" lecture by being assigned an EBP mentor who helps them appraise and assimilate published literature.

\section{Instrument Development}

\section{Conceptual Model}

Our conceptual model of EBP expertise was developed during a series of meetings of the investigators, local EBP experts, and clinical educators. It held that with increasing education in EBP, practitioners would 1) report increasing comfort with EBP techniques (comfort with EBP); 2) have increasing self-efficacy with the use of EBP concepts and methods (self-reported practice of EBP); and 3) show measurably improved knowledge and skill in using the concepts of EBP to solve realistic patient problems (knowledge).

\section{Item Development}

We wrote demographic, comfort level, and self-reported practice of EBP questions based upon our conceptual model. Based on the social cognitive theory of self-efficacy, questions concerning comfort level and self-reported practice of EBP are important as they are likely to correlate with actual behavior. If a person perceives himself/herself to be capable of performing in a certain manner, then he/she is more likely to attain that goal. ${ }^{10}$ For these questions, we relied heavily on unpublished questions developed by Dr John Frohna when at the University of Michigan (J. Frohna, personal communication, July 2006). He used these questions as the tool to assess his residents' comfort level and self-reported practice of EBP, both before and after evidence-based medicine (EBM) teaching sessions. For the knowledge portion of the instrument, we developed items according to a content map based on our residency program's curriculum and a core text. ${ }^{9}$ Although core texts on EBP usually specify 4 major types of questions, we focused on diagnosis and therapy as being most relevant to general pediatricians. Ideas for questions also came from previous validated questionnaires like the Fresno and Berlin questionnaires. ${ }^{6,7}$ We wrote openended questions to ensure a higher order of thinking. ${ }^{6}$

Local EBP experts revised each draft by using the Delphi method. ${ }^{11}$ According to their feedback, we eliminated or edited items to decrease ambiguity and to ensure content validity. We developed scoring criteria for the knowledge items based upon key words and correct calculations. Most questions scored out of 5 points, with partial credit given for incomplete answers.

\section{Instrument Description}

The instrument divides into 4 parts: demographics, comfort level, self-reported practice of EBP, and EBP knowledge. The section on basic demographic information asks about the participant's age, gender, previous education, and prior EBP exposure. The comfort level section asks 6 Likert-type items, such as comfort in one's ability to generate a clinical question, access a computer database like Medline, or critically appraise an article dealing with a new therapeutic intervention (Figure 1). The section on self-reported practice of EBP asks 7 items, such as how often one searches articles to answer a clinical question or generates clinical questions applicable to his or her patient's diagnostic or therapeutic plan.

The final section, which is also the bulk of the instrument, is EBP knowledge. There are 10 constructed response questions. We wrote 2 versions of the questions that tested identical concepts. Having 2 knowledge question sets allows us to administer the assessment twice without the user being able to rely on superficial similarity. In the validation study, each set was done by half of the participants.

The 10 questions are organized around 2 pediatric clinical scenarios: a "therapy" scenario (eg, which drug to use in asthma or which rehydration therapy to use for gastroenteritis) and a "diagnosis" scenario (eg, which diagnostic test to use for a urinary tract infection or which screening criteria to use to predict serious bacterial illness in babies).

For the therapy scenario, the participant must answer where one would search for the best evidence, how to form a searchable question, and what is the best type of study design to answer the clinical question. Then, 2 abstracts from the medical literature are provided. The participant must answer which abstract better answers the question and, based on the abstract, what is the number needed to treat (ie, to prevent one hospitalization). We believe that using published abstracts requires trainees to understand the validity of the published study and its relevance to the care of his or her patient. All abstracts are credited to their respective authors. Subjects are also asked to define key EBP concepts in both the therapy and diagnosis scenarios.

For the diagnosis scenario, the participant must answer again where one would search for the best evidence, how to form a searchable question, and what is the best type of study design to answer the clinical question. They are then provided with an abstract and asked the probability that the presented patient has the disease based on the pretest likelihood, the likelihood ratios, and the provided Fagan nomogram.

\section{Validation}

\section{Subjects}

The subjects for the validation study represented 4 levels of expertise: 


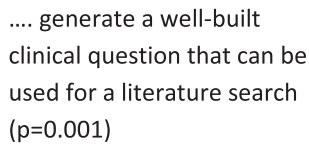

...critically appraise an article dealing with a new therapeutic intervention $(p=0.000)$

...critically appraise an article dealing with a new diagnostic intervention $(p=000)$

...teach evidence-based
medicine concepts to my
peers
$(p=0.000)$

1
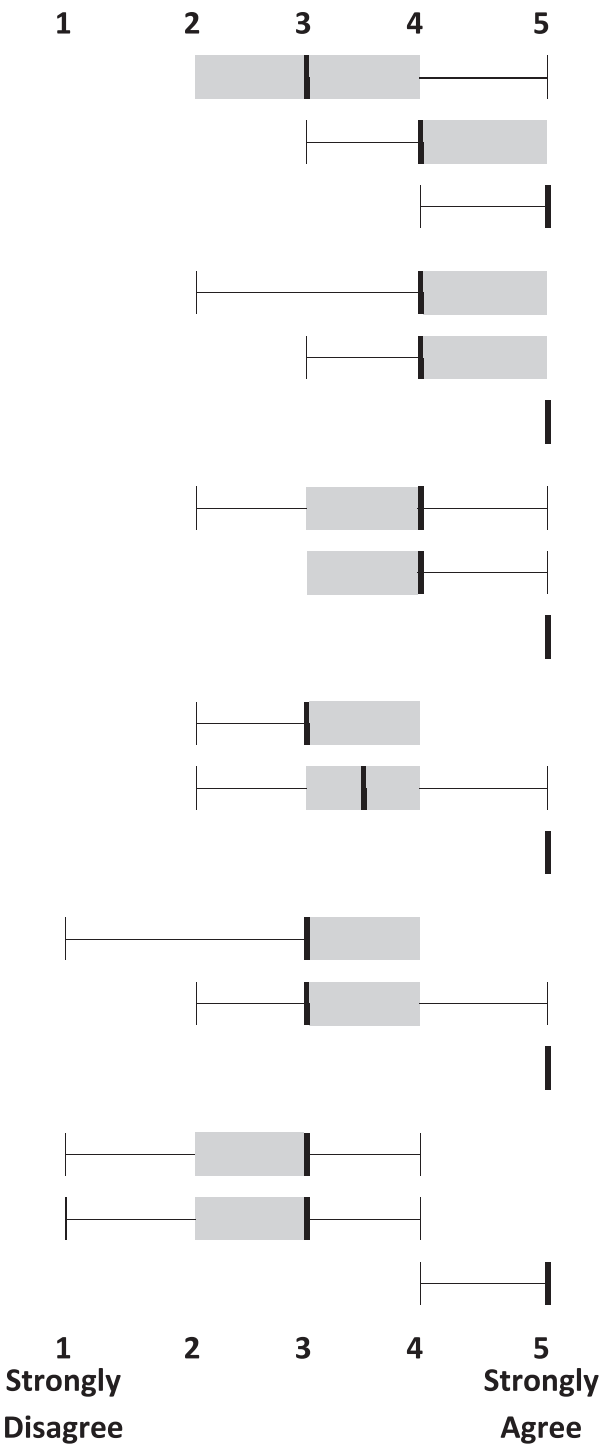

Figure 1. Comfort level of subjects with evidence-based practice. Box and whisker plots of Likert-type responses of subjects to 6 questions about their comfort with evidence-based practice (EBP). The darkest vertical line is the median response. Left and right box edges are the 25th and 75th percentiles, respectively. The whiskers represent the fifth and 95th percentiles. For any one question, there are 3 sets of plots: preclinical medical students (top), pediatric interns and residents (middle), and EBP experts (bottom). $\mathrm{P}$ values refer to overall $3 \times 5$ chi-square.

1. Preclinical medical students (MS-2). These were medical students who had just completed their second year of medical school at the Columbia University School of Medicine. They had not yet participated in any clinical clerkships nor received any formal training in either pediatrics or evidence-based medicine.

2. Incoming pediatric interns (PGY-1). These were graduated medical students who were PGY-1s at the start of intern orientation for their pediatric residency at our hospital. All interns were likely to have had some prior EBP exposure and experience in pediatrics during their clinical clerkships.

3. Second- and third-year pediatric residents (PGY2-3). These were pediatric residents who were about to start second and third years of residency. They completed the instrument toward the end of our year-long EBM curriculum.
4. EBP experts (experts). These experts were part of the Teaching Evidence-Based Emergency Medicine Workshop held at the New York Academy of Medicine in April 2007. Each specialized in pediatrics, emergency medicine, or both.

\section{Procedure}

To assess whether our instrument is reliable and valid, we aimed for it to have certain properties. These included internal consistency, interrater reliability, content validity, acceptable item difficulty, and construct validity.

From March 2007 to June 2007, we randomly distributed a version of the instrument to all 4 groups: MS-2, PGY-1, PGY2-3, and EBP experts. We included both incoming second- and third-year residents in one group because the curriculum in our residency program began formally in July 2006, and our group considered these residents to have similar prior EBP education. 
MS-2s completed the instrument over a 45-minute period in a proctored setting during an introduction to pediatric clerkship workshop. PGY-1s completed the instrument during pediatric intern orientation, also in a proctored setting. PGY2-3s and experts were handed the instrument and completed it on their own time without proctoring. Instruments were completed anonymously.

We asked the participants to report their prior EBP formal education: none, previous participation in an EBM session that was less than 1 day ( $<1$ day), previous participation in an EBM workshop that was more than 1 day $(>1$ day), and those who have a Masters of Public Health or have already served as a tutor, facilitator, or trainer in another EBM workshop (tutor). Scoring was done by one of the investigators (Lauren Chernick or Heather Liu) according to the scoring scheme. The other scorer rescored a subset of items $(20 \%)$ to determine interrater reliability.

\section{Statistical Analysis}

Descriptive analyses were performed to examine the demographics of the subjects. Comfort level and selfreported practice of EBP were analyzed using descriptive statistics, including Tukey box plots. For the EBP knowledge items, internal reliability of the tests was reported using Cronbach's $\alpha .{ }^{12}$ We considered Cronbach's $\alpha>.7$ as satisfactory. ${ }^{13}$ We determined the interrater agreement by using Cohen's $\kappa .{ }^{14}$ Floor and ceiling effects were determined by qualitatively examining frequency plots of individual item scores. Knowledge item difficulty was calculated using the proportion correct across all subjects. Construct validity was estimated by determining whether the test items could discriminate between groups with varying levels of professional experience and prior education in EBP by using 1-way analysis of variance (ANOVA). We determined the relationship of the 3 subscales to one another by using Pearson's correlation coefficient as well as by performing linear regression. For the linear regression, we verified the assumptions of linearity, independence of errors, homogeneity of variance, and normality of error distribution. All scores are reported as \pm SD except where noted. All analyses were performed using SPSS version 11 (SPSS Inc, Chicago, Ill).

\section{RESULTS}

\section{Validation}

\section{Demographics}

We approached 13 MS-2s, 15 PGY-1s, 39 PGY2-3s, and 10 experts. Of those 77, $56(73 \%)$ subjects completed the instrument. This consisted of 13 of 13 (100\%) MS-2s, 13 of $15(87 \%)$ pediatric interns, 22 of $39(56 \%)$ pediatric residents, and 8 of $10(80 \%)$ experts.

Of all 56 participants, $12(21 \%)$ reported no prior EBP experience, $25(45 \%)$ reported less than 1 day of prior EBP education, $12(21 \%)$ reported more than 1 day of prior EBP experience, and $7(13 \%)$ reported to be an EBP tutor. Based on professional level, 11 of 12 (92\%) MS-2s reported no prior EBP experience, whereas $1(8 \%)$ reported less than 1 day of EBP education. Of the 13 incoming interns, $10(77 \%)$ reported less than 1 day of prior EBP education, whereas 3 (23\%) reported more than 1 day. Of the 22 pediatric residents, 1 (5\%) reported no prior EBP experience, $13(59 \%)$ reported less than 1 day, and 8 (36\%) reported more than 1 day. Seven of 8 experts reported to be EBP tutors.

\section{Comfort Level}

Using a Likert scale, comfort level ( 1 = strongly disagree, $5=$ strongly agree, \pm SD) increased with professional level (MS-2: $2.8 \pm 0.7$; PGY-1: $3.6 \pm 0.5$; PGY2-3: $3.6 \pm 0.7$; expert: $4.9 \pm 0.1$ ). However, there was no difference in comfort level between incoming interns and PGY2-3s. Comfort level did increase between all groups based on prior EBP education (none: $2.9 \pm 0.8 ;<1$ day: $3.5 \pm 0.6$; $>1$ day: $3.9 \pm 0.8$; tutor: $4.9 \pm 0.1$ ). The overall mean was $3.6 \pm 0.9$.

\section{Self-Reported Practice of EBP}

Self-reported practice of EBP was also based on a Likert scale $(1=$ never, $5=$ always, $\pm \mathrm{SD})$. Self-reported practice increased with professional level, except between PGY-1s and PGY2-3s (MS-2: $2.0 \pm 0.70$; PGY-1: $3.1 \pm 0.7$; PGY2-3: $3.0 \pm 0.6$; experts: $3.6 \pm 0.6$ ). Self-reported practice of EBP increased with prior EBP education (none: 2.1 $\pm 0.8 ;<1$ day: $2.8 \pm 0.7 ;>1$ day: $3.2 \pm 0.5$; tutor: $3.7 \pm$ 0.6 ). The overall mean was $2.8 \pm 0.8$.

\section{Knowledge}

The 2 versions of each of the knowledge questions performed similarly in all analyses. The knowledge subscale is reliable. For internal consistency, the Cronbach's $\alpha=.80$. Item test correlations were all positive and $>0.2$. Dropping any 1 item did not appreciably improve the reliability. Interrater reliability was very good, with $\kappa=0.94$. None of the knowledge items showed floor or ceiling effects. With the maximum number of points per question being 5 , the overall mean for all items was $2.7 \pm 1.0$ (minimum score $=1.1$, maximum score $=4.1$ ). Average score on the knowledge subscale was similar between version 1 (27.7) versus version 2 (26.0). The $95 \%$ confidence interval for the difference, adjusted for professional level, was -0.4 to 5.2, favoring version 1 .

We showed that those participants at a higher professional level and those with increasing prior EBP experience had higher EBP scores on the EBP knowledge part of the instrument (Figures 2 and 3). Scores on the knowledge section (out of $50 \pm \mathrm{SD}$ ) rose with training level (MS-2: $14.8 \pm$ 5.7; PGY-1: $22.2 \pm 3.4$; PGY2-3: $31.7 \pm 6.1$; experts: $43 \pm 4.0$; ANOVA, $P<.001)$. All Bonferroniadjusted subgroup pairwise comparisons were statistically significant. We found identical results for prior EBP education as the predictor variable. The validity properties of our instrument are summarized in the Table.

\section{Overall}

The comfort subscale and the self-reported practice of EBP subscale were correlated with each other $\left(r^{2}=0.36\right.$; 


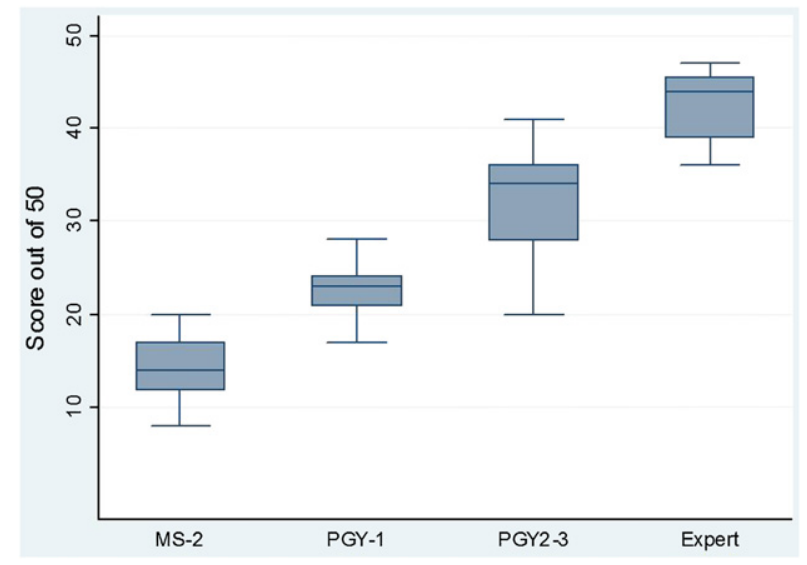

Figure 2. Total evidence-based practice (EBP) score by professional level. Box plots show mean score (middle line) with 25 th/75th percentiles (box edges) and fifth/95th percentiles (whiskers). Y-axis is raw score on the knowledge test items of the scale. Maximum score is 50 points. Overall analysis of variance, $\mathrm{P}<.001 . M S-2=$ second-year medical students; $P G Y-1=$ pediatric interns; $P G Y 2-3=$ second- and third-year pediatric residents; expert $=$ attending physician with EBP expertise.

$P<.001)$ and with EBP knowledge score $\left(r^{2}=0.49\right.$, $P<.001$ and $r^{2}=0.39, P<.001$, respectively). Linear regression showed that the relationship between the comfort scale and knowledge score remained significant even after adjustment for professional level.

\section{DISCUSSION}

The ACGME Outcome Project stresses the importance of the identification and development of measurement tools to help educators evaluate the outcomes of their educational efforts. ${ }^{3}$ By the end of the Project's phase 3 in 2011, postgraduate training programs will be held rigorously accountable to the ACGME for ensuring the proficiency of their residents in these areas. The development of reliable, valid instruments will bring us closer to achieving this larger goal.

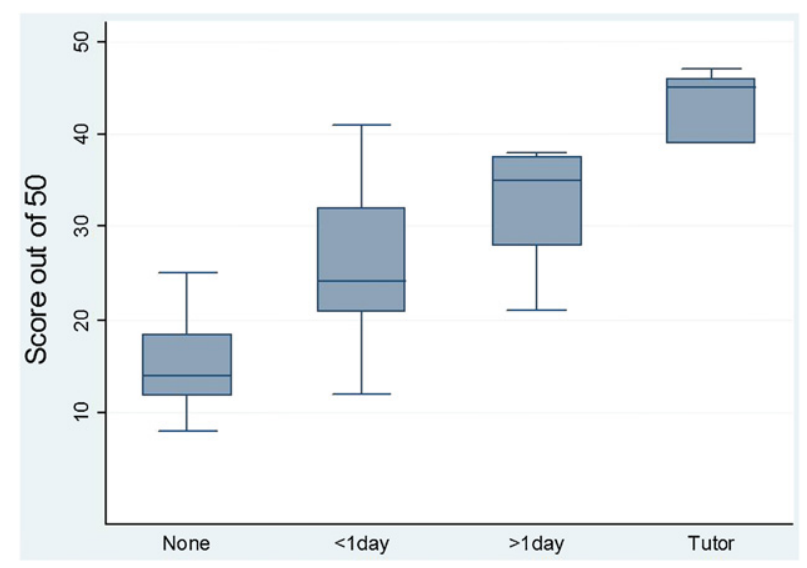

Figure 3. Total evidence-based practice (EBP) score by prior EBP experience. Box plots show mean score (middle line) with 25 th/75th percentiles (box edges) and fifth/95th percentiles (whiskers). Y-axis is raw score on the knowledge test items of the scale. Maximum score is 50 points. Overall analysis of variance, $\mathrm{P}<.001$.
Our conceptual framework is likely to be practically relevant to pediatric educators. In addition to describing how much their trainees know based on realistic pediatric scenarios, the instrument attempts to measure their selfefficacy with these principles as well as document their self-reported EBP behaviors, such as their propensity to do a literature search for a question arising from the care of one of their patients.

We developed an instrument that is reliable and valid for assessing resident knowledge in EBP. The groups we used to validate this instrument show a range in proficiency in EBP. By including 4 groups of varying levels, we showed that the knowledge component of the instrument can differentiate levels of expertise across the medical education spectrum. We are confident that our scale can distinguish intern-level knowledge from that of senior residents. A knowledge scale with finer discrimination would require calibration on a much larger resident pool, likely on a multi-institutional level.

We also found that comfort with EBP and self-reported practice of EBP correlated to a reasonable level with participant's performance on the knowledge section, except between incoming interns and senior residents. Their comfort and self-reported practice scores were similar. Residents completing a year of EBP curriculum felt and rated their skills as poorly as those without such EBP training, even though their knowledge section scores were higher.

Although we do not suggest that the comfort or selfreported practice of EBP components be used by educators to replace direct assessment of EBP knowledge, we do believe that these subscales correlate to the knowledge level of novices, residents (PGY-1 and PGY2-3), and experts. Since the 6 questions of the comfort subscale and 7 questions of the self-reported practice are easily administered, they can be a quick screen of the learners before the educational intervention ensues and could help educators differentiate those residents that are at the level of novice and expert.

Our suggestion would be for EBP educators to use this instrument as a means to understand the proficiency of pediatric residents in EBP, both before and after curriculum interventions. This way educators can gauge the success of their educational interventions. We have submitted the instrument for publication on MedEdPORTAL so that it can be accessible to pediatric educators. ${ }^{16}$

There were limitations to our study. The EBP content we included is what our group found to be most clinically relevant (ie, therapy and diagnosis); however, there are certain EBP skills that our instrument did not assess (ie, harm and prognosis). The completion of the instrument was not proctored for PGY2-3 and experts. It is also noted that pediatric residents claimed different levels of prior EBP education. Since the investigators of this study knew that all of these residents participated in weekly lectures, there may have been ambiguity in the way we asked about prior EBP education. A threat to the generalizability of this study is the fact that our pediatric residency program placed a special emphasis on EBP education during the year prior 
Table. The validity properties of our pediatrics-based instrument assessing knowledge of evidence-based practice*

\begin{tabular}{|c|c|c|c|}
\hline Test Property & Measure Used & Acceptable Results & Performance of Our Test \\
\hline \multicolumn{4}{|l|}{ Reliability properties } \\
\hline Internal consistency & Cronbach's $\alpha$ & $>.6$ & .80 \\
\hline Interrater reliability & Interrater correlation & $\kappa>0.4$ & $\kappa=0.94$ \\
\hline \multicolumn{4}{|l|}{ Validation properties } \\
\hline Content validity & Expert opinion (Delphi method) & Test covers the main topics of EBP $\dagger$ & $\begin{array}{l}\text { Covers the topics in our EBP } \\
\text { curriculum }\end{array}$ \\
\hline Item difficulty & Proportion correct & No floor or ceiling effects & $\begin{array}{l}\text { Minimum mean }=1.13 \\
\quad \text { Maximal mean }=4.09\end{array}$ \\
\hline Construct validity & Mean scores of experts and novices & $\begin{array}{l}\text { Higher scores with increasing } \\
\text { professional level and prior } \\
\text { EBP experience (ANOVA) } \$\end{array}$ & $\begin{array}{l}\text { Higher EBP scores correlated with } \\
\text { increasing professional level } \\
\left(r^{2}=0.76\right) \text { and prior } \\
\text { EBP experience }\left(r^{2}=0.6\right)\end{array}$ \\
\hline
\end{tabular}

*Adapted from Cook and Beckman ${ }^{15}$ and Ramos and colleagues. ${ }^{6}$

$\dagger \mathrm{EBP}=$ evidence-based practice.

$\$ \mathrm{ANOVA}=$ analysis of variance.

to the study period. Other programs with more or less of an EBP culture may get different results.

Of all pediatric residents approached to participate, $35 \%$ (19 of 54) did not complete the instrument; this may have led to selection bias since those who completed the instrument may have felt more comfortable with their skills than those who did not complete it. Yet, we think these numbers are acceptable, as the completion of the instrument by 13 PGY-1s and 22 PGY2-3s is comparable to sample sizes of validation studies in other specialties. ${ }^{5}$ Furthermore, this is the first validation study to our knowledge that focuses specifically on EBP and pediatric residents.

We had 2 versions of the EBP knowledge subscale so that the instrument could be administered more than once to a given trainee. In our validation, each individual completed only one of the versions, so that we were unable to assess parallel forms reliability. However, the scenarios performed almost identically in our other analyses (reliability, difficulty, and correlations with other subscales). Scores between the 2 versions cannot be directly compared, as the scores on version 1 may be higher. Having 2 sets of questions allows the educator the flexibility of administering the instrument to a given group of learners a second time to assess their progress.

The following definition items presented difficulty in scoring: allocation concealment, bias, randomization concealment, and masking. We used a third scorer to resolve any conflict. Finally, in our instrument we provided abstracts for the participants to appraise, which might limit our capability to fully evaluate the participants' ability to appraise the methodology of the full text of such studies.

\section{Conclusion}

We have developed and validated a reliable instrument to assess resident proficiency in EBP. To our knowledge, this is the first validated instrument, based in pediatric content, specifically designed for pediatric residents. Benefits of the instrument include helping pediatric EBP educators evaluate residents over space and time and having a self-assessment component. The knowledge component of the instrument can also differentiate levels of learners. Further, the instrument can gauge competency of residents in attaining EBP skills after participating in a formal EBP curriculum.

\section{ACKNOWLEDGMENT}

The authors thank Dr John Driscoll for his support in the development of an EBP curriculum in our residency program.

\section{REFERENCES}

1. Sackett DL, Rosenberg WM, Gray JA, et al. Evidence-based medicine: what it is and what it isn't. BMJ. 1996;312:71-72.

2. Frohna JG, Park SM. Promoting the use of evidence-based medicine in pediatrics. $J$ Pediatr. 2002;141:599-600.

3. Accreditation Council for Graduate Medical Education. ACGME Outcome Project. Available at: http://www.acgme.org/outcome/ comp/GeneralCompetenciesStandards21307.pdf. Accessed April 23, 2010.

4. Academic Pediatric Association. Educational guidelines for pediatric residency. Available at: http://www.ambpeds.org/egwebnew/. Accessed August 22, 2008.

5. Shaneyfelt T, Baum KB, Bell D, et al. Instruments for evaluating education in evidence-based practice-a systematic review. JAMA. 2006;296:1116-1127.

6. Ramos K, Schafer S, Tracz S. Validation of the Fresno test of competence in evidence based medicine. BMJ. 2003;326:319-321.

7. Fritsche L, Greenhalgh T, Falck-Ytter Y, et al. Do short courses in evidence based medicine improve knowledge and skills? Validation of Berlin Questionnaire and before and after study of courses in evidence-based medicine. BMJ. 2002;325:1338-1341.

8. Gruppen LD. Implications of cognitive research for ambulatory care education. Acad Med. 1997;72:117-120.

9. Guyatt GH, Rennie D, eds. Users' Guides to the Medical Literature: A Manual of Evidence-Based Clinical Practice. Chicago, Ill: AMA Press; 2002.

10. Bandura A. Self-efficacy: toward a unifying theory of behavioral change. Psychol Rev. 1977;84:191-215.

11. Brown B. Delphi Process: A Methodology Used for the Elicitation of Opinions of Experts. Santa Monica, CA: RAND Document No: P$3925 ; 1968$.

12. Cronbach LJ. Coefficient alpha and the internal structure of tests. Psychometrika. 1951;16:297.

13. Kline P. Handbook of Psychological Testing. London, England: Routledge; 2000.

14. Jacob Cohen. A coefficient of agreement for nominal scales. Educ Psychol Meas. 1960;20:37-46.

15. Cook D, Beckman T. Current concepts in validity and reliability for psychometric instruments: theory and application. Am J Med. 2006; 119:166.e7-e16.

16. Association of American Medical Colleges. MedEdPORTAL. Available at: http://www.mededportal.org. Accessed May 10, 2010. 